\title{
Aspergilosis invasora multisistémica en un niño inmunocompetente. Comunicación de un caso
}

\author{
Alejandro Donoso F., Jorge Camacho A., Pedro Alarcón L. y Pablo Cruces R.
}

\section{Invasive multisystemic aspergillosis in an immunocompetent child. Case report}

Invasive aspergillosis is a severe disease, with an increased incidence in last decades. It occurs mainly in immunocompromised patients and rarely affects immunocompetent hosts. We describe a previously healthy 8 -year-old boy who acquired an invasive aspergillosis of abdominal origin associated with an opened laparotomy; within three weeks the patient progressed to a multiple organic failure syndrome and died despite amphotericin B deoxycolate plus voriconazole treatment and aggressive surgical debridement. Necropsy revealed multiple parenchyma involvement with characteristic findings of angioinvasive aspergillosis. Invasive aspergillosis must be considered an emergent infection in critical pediatric patients, including previously immunocompetent hosts; it is associated with high mortality despite adequate and opportune therapy.

Key words: immunocompetent, invasive aspergillosis, Aspergillus fumigatus, pediatrics.

Palabras claves: inmunocompetente, aspergillosis invasora, Aspergillus fumigatus, pediatría.

\section{Introducción}

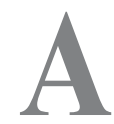
spergillus es un hongo filamentoso hialino de amplia distribución. Existen aproximadamente doscientas especies conocidas, pero sólo unas pocas son patógenas para el ser humano, siendo la más común Aspergillus fumigatus. La infección habitualmente ocurre por inhalación del conidio, causando una variedad de síndromes clínicos, desde un aspergiloma hasta la aspergilosis invasora (AI) fulminante ${ }^{1}$.

La AI es una infección oportunista grave, que habitualmente afecta a huéspedes inmunocomprometidos, como pacientes oncológicos, sometidos a trasplante de órganos, portadores de enfermedad granulomatosa crónica y SIDA ${ }^{2-4}$. Tiene una elevada letalidad a pesar de un óptimo tratamiento. La presencia de neutropenia durante un período prolongado es el principal factor de riesgo conocido para el desarrollo de $\mathrm{AI}^{4,5}$, aunque también lo son la prematurez extrema y el uso frecuente de corticosteroides ${ }^{6,7}$. Rara vez la AI ocurre en individuos inmunocompetentes ${ }^{8}$, en quienes afecta predominantemente el parénquima pulmonar.

Comunicamos el caso de un escolar con 8 años de edad, inmunocompetente, que desarrolló una AI multisistémica con sitio de inicio abdominal en relación a una laparotomía contenida, evolucionando hacia una falla orgánica múltiple, con un desenlace fatal, a pesar del tratamiento empleado.

\section{Caso clínico}

Paciente de 8 años, sexo masculino, operado de peritonitis apendicular seis meses antes, cuya histología mostró un apéndice de aspecto isquémico con perforación puntiforme en su tercio distal; sin otros antecedentes mórbidos de importancia. Consultó a su hospital de origen por dolor periumbilical progresivo y 3 días de evolución, vómitos biliosos, fiebre $39^{\circ} \mathrm{C}$, anorexia y compromiso del estado general, cuadro interpretado como una obstrucción intestinal complicada y shock séptico secundario. Durante el acto quirúrgico se demostró obstrucción por bridas, con perforación y necrosis de ileon distal y peritonitis secundaria, realizándose una resección ileal de $20 \mathrm{~cm}$ y laparotomía contenida. Al tercer día post operatorio fue derivado a nuestro centro por persistir en anuria y necesitar de terapia de reemplazo renal. Al ingreso estaba en shock séptico descompensado, por lo que requirió soporte vasoactivo con epinefrina. Se inició inmediatamente hemofiltración veno-venosa continua y monitorización invasora, antibioterapia con ampicilina, cefotaxima y metronidazol, y nutricional parenteral total. A las 24 horas se realizó laparotomía exploradora, aseo, quedando nuevamente con laparotomía contenida. En una re-exploración al día siguiente se efectuó resección de $10 \mathrm{~cm}$ de ileon necrótico. Requirió terapia con infusión de insulina

\author{
Hospital Padre Hurtado. \\ Área de Cuidados Críticos. \\ Unidad de Gestión Clínica del Niño \\ (ADF, JCA). \\ Laboratorio Microbiología (PAL) \\ Universidad del Desarrollo- \\ Clínica Alemana \\ Programa de Medicina Intensiva \\ Infantil (PCR). \\ Recibido: 28-06-05 \\ Aceptado: 24-11-05 \\ Correspondencia a: \\ Alejandro Donoso Fuentes \\ adonoso@hurtadohosp.cl
}




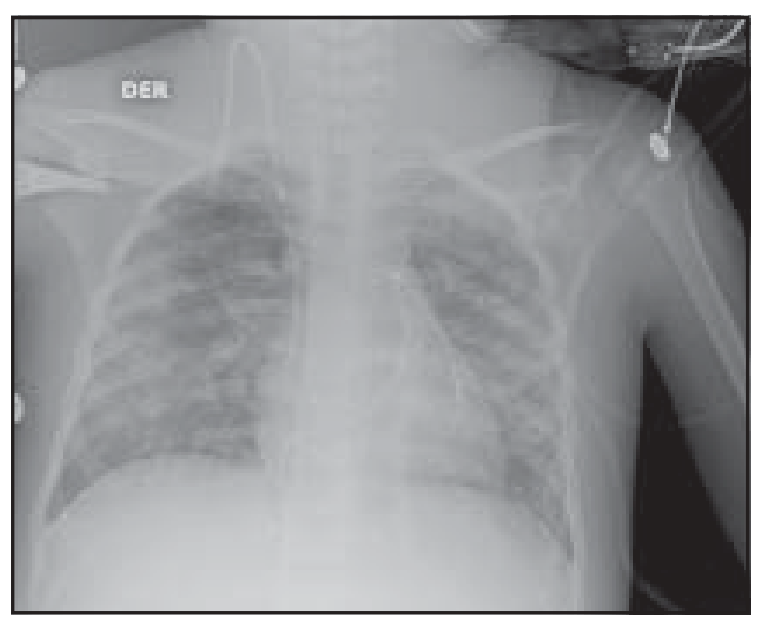

Figura 1. Radiografía de tórax (PA) de paciente de 8 años cursando con síndrome de distress respiratorio agudo por aspergilosis invasora multisistémica. En el campo pulmonar izquierdo se visualizan imágenes nodulares.

durante siete días en dosis de $0,02-0,03 \mathrm{U} / \mathrm{kg} / \mathrm{h}$ por hiperglicemia mantenida sobre $200 \mathrm{mg} / \mathrm{dl}$. Tras una semana, se logró su estabilización hemodinámica, pudiendo suspenderse el apoyo vasoactivo e insulina. Se mantuvo en ventilación mecánica con parámetros mínimos para el manejo de su laparotomía contenida, estando asintomático respiratorio y persistiendo en anuria. Al noveno día se cerró la laparotomía; sin embargo, la ileostomia presentó aspecto necrótico con una coloración verde, cuyo cultivo fue positivo para A. fumigatus. Dos días después se decidió efectuar lavado bronco alveolar por secreciones bronquiales purulentas y aparición de nódulos pulmonares (Figura 1), de cuyo cultivo también fue aisló A. fumigatus. El diagnóstico micológico fue certificado posteriormente en el Laboratorio de Micología del Programa de Microbiología y Micología, Instituto de Ciencias Biomédicas, Facultad de Medicina, Universidad de Chile. Inició entonces terapia antifúngica con anfotericina B deoxicolato (1 mg/ $/ \mathrm{kg} /$ día) y voriconazol (carga: $25 \mathrm{mg} / \mathrm{kg}$ ev, mantención: $7 \mathrm{mg} / \mathrm{kg} / \mathrm{d}$ ía ev), lográndose negativizar los cultivos traqueal y de ostomías en controles ulteriores. La cuantificación de inmunoglobulinas séricas estuvo dentro de rango normal y el test de ELISA para VIH fue negativo. En los días siguientes continuó en anuria y persistió febril, a pesar del esquema empleado $y$ el drenaje de colecciones en la pared abdominal (tenía una TAC de abdomen normal). Se amplió empíricamente la antibioterapia adicionando imipenem. En la tercera semana, presentó inestabilidad hemodinámica, hiperlactacidemia y evidencias de SDRA y de shock séptico; por distensión abdominal progresiva se exploró quirúrgicamente en UCI dadas las condiciones del paciente, drenándose pequeñas colecciones purulentas de la pared abdominal y de la zona retrovesical; quedó una vez más con laparotomía contenida. En las horas siguientes hubo franco deterioro hemodinámico $\mathrm{y}$ ventilatorio, refractario a aminas vasoactivas, falleciendo al vigésimo cuarto día de internación. Un test de galactomanano en sangre tomado el día previo al fallecimiento fue negativo. Alcanzó a completar un total de 15 días con anfotericina B y voriconazol.

El estudio anátomo-patológico reveló la presencia de hifas características de Aspergillus sp dentro de diversos parénquimas, con invasión vascular: encéfalo, pulmón, corazón, tubo digestivo, páncreas, hígado y riñones, lo cual es compatible con el diagnóstico de aspergilosis angioinvasora sistémica, y signos de falla orgánica múltiple.

\section{Discusión}

La incidencia de AI se ha incrementado en las últimas décadas, en forma paralela al aumento del número de pacientes inmunodeprimidos por quimioterapia o uso de inmunosupresores post transplante ${ }^{5}$. Datos del CDC de Atlanta, E.U.A., revelan que la mortalidad asociada a AI ha experimentado un aumento de casi $400 \%$ desde $1980^{\circ}$.

Aunque el primer reporte en pediatría se remonta a $1936^{10}$, en opinión de diversos autores la verdadera incidencia de AI en la población pediátrica es desconocida.

Aspergillus sp es un hongo ambiental presente en la tierra y en el polvo, su principal ruta de transmisión es aérea y la penetración al organismo humano primariamente respiratoria. Así, la inhalación de las conidias habitualmente precede a la enfermedad invasora. Es conocido el papel que juega en su adquisición nosocomial la contaminación del aire cuando se ejecutan labores de construcción en el hospital o en su vecindad, como también cuando se contaminan los sistemas de ventilación. Habitualmente el compromiso inicial afecta a las cavidades paranasales y pulmones, pero cerca de $25 \%$ desarrolla una infección sistémica, aunque esta cifra variará según la población estudia$\mathrm{da}^{11}$. En niños han sido comunicados también como puerta de entrada sitios de trauma y de inserción de líneas venosas ${ }^{12,13}$.

En el caso de nuestro paciente, la evolución cronológica de los eventos nos hace plantear a modo hipótesis que la probable "puerta de entrada" para la AI fue la presencia de la laparotomía contenida, dado que al momento del diagnóstico no existía evidencia alguna de compromiso pulmonar clínico ni radiológico. La contaminación e infección de heridas quirúrgicas por Aspergillus ha sido comunicada con anterioridad ${ }^{14}$.

La evaluación radiológica del tórax es la base para la sospecha diagnóstica de una AI, así en series de adultos hasta $50 \%$ de los casos presentaban cavitación y 
$40 \%$ la formación de crescéntica aérea. Se señala que hay concordancia entre el tipo de hallazgo radiológico y la edad del paciente, siendo estos signos más frecuentes de observar en niños mayores y adultos ${ }^{15-17}$. El uso de TAC de tórax de alta resolución ha desplazado a la radiología convencional en el diagnóstico de aspergilosis pulmonar ya que permite la visualización de lesiones en forma más precoz y una mayor definición del tipo de lesiones mejorando francamente el rendimiento de la radiografía ${ }^{18}$.

El diagnóstico definitivo requiere la visualización de hifas asociadas a daño tisular en un análisis histopatológico o la existencia de un cultivo positivo asociado a evidencias clínicas o radiológicas de infección ${ }^{19}$. La disponibilidad actual de nuevos elementos de ayuda como el test antigénico (galactomanano), debe de ser considerada ${ }^{20}$, el que ha sido aprobado para uso en adultos, pero presenta frecuentes falsos positivos y negativos en la población infantil, como ocurrió en nuestro paciente.

Existen variados factores de riesgo para el desarrollo de AI, siendo el principal la magnitud y duración de la neutropenia, en especial si ella es menor a 100 neutrófilos $/ \mathrm{mm}^{3}$, ya que el leucocito neutrófilo junto a macrófagos conforman la primera línea de defensa contra Aspergillus sp. Es conocido que los pacientes con una estadía prolongada en UCI, como el aquí presentado, experimentan un deterioro en su función inmune (estado hipoinmune) en el contexto de un síndrome compensatorio de respuesta antiinflamatoria. Así entonces, este estado de inmunoparálisis puede contribuir a la adquisición de infecciones fúngicas en pacientes sin otras condiciones predisponentes más que una disfunción orgánica múltiple ${ }^{21,22}$. Por otra parte, la existencia de hiperglicemia es conocida como deletérea para la función inmune de los neutrófilos periféricos ${ }^{23}$. Otro factor predisponerte habitualmente señalado en diversas comunicaciones es el uso de corticoesteroides $^{7,24}$.

Con respecto al desarrollo de $\mathrm{AI}$ en huéspedes inmunocompetentes, existen variados reportes asociados a diversas patologías como influenza, infecciones rino-sinusales, infección de herida operatoria, etc., aunque ninguno de ellos en un paciente pediátrico con un compromiso tan extenso como el aquí comunicado $^{8,14,25-27}$.

El tratamiento de la AI tiene tres pilares fundamentales, a saber: el uso de antifúngicos (profiláctico, empírico o ante infección documentada), el drenaje quirúrgico y la reversión de la condición predisponente. En pacientes sin inmunocompromiso, gran parte del éxito de la terapia empleada depende un diagnóstico precoz ${ }^{28-33}$. Hay concordancia que el compromiso del SNC es generalmente fatal.
El tratamiento antifúngico convencional hasta ahora ha sido anfotericina $\mathrm{B}$, con el que se ha documentado una sobrevida de alrededor de $50 \%$, sin embargo, su uso sobre todo en altas dosis (sobre $1 \mathrm{mg} / \mathrm{kg} / \mathrm{día}$ ) se asocia a nefrotoxicidad, por lo que se han desarrollado formulaciones lipídicas, menos tóxicas.

Un nuevo antifúngico, voriconazol, ha sido validado en el tratamiento primario de aspergilosis invasora en adultos, obteniendo una mejor eficacia clínica y sobrevida comparado con anfotericina $\mathrm{B}^{34}$. Su uso es promisorio además, por su habilidad para cruzar la barrera hematoencefálica y también su excelente absorción oral y biodisponibilidad ${ }^{35}$. Caspofungina se ha utilizado en protocolos de rescate en pacientes adultos con aspergilosis invasora con intolerancia o refractarios al tratamiento con anfotericina $\mathrm{B}^{36}$. Además se ha reportado en forma anecdótica el uso de asociaciones de antifúngicos (anfotericina B más voriconazol, voriconazol más caspofungina, etc) con buenos resultados clínicos pero aún no validadas por estudios controlados. Estos nuevos fármacos se encuentran en investigación en pediatría para conocer su farmacocinética y dosis óptimas en niños ${ }^{37,38}$.

El tratamiento quirúrgico esta orientado a la resección del tejido infectado o necrótico y es frecuente de emplear en los casos de sinusitis y ante la existencia de lesiones cutáneas.

Probablemente el fracaso de la terapia antifúngica en nuestro paciente se debió en parte a la imposibilidad de revertir la inmunoparálisis y a la presencia de focos infecciosos no resecables.

\section{Resumen}

La aspergilosis invasora es una patología grave, con una incidencia en aumento, que generalmente afecta a pacientes con inmunosupresión, existiendo escasa información en pacientes pediátricos inmunocompetentes. Reportamos el caso de un escolar de 8 años, previamente sano, que cursó con una aspergilosis invasora multisistémica de origen abdominal, en relación a una laparotomía contenida, evolucionando hacia una falla orgánica múltiple, con un desenlace fatal, a pesar de recibir terapia con anfotericina B deoxicolato más voriconazol y drenaje quirúrgico amplio. El estudio anátomo-patológico reveló elementos de hifas características dentro de diversos parénquimas e invasión vascular. La aspergilosis invasora debe tenerse presente entre las causas de infecciones emergentes en pacientes críticos, incluso en inmunocompetentes; se asocia a una elevada mortalidad a pesar de un adecuado y oportuno tratamiento. 


\section{Referencias}

1.- Soubani A O, Chandrasekar P H. The clinical spectrum of pulmonary aspergillosis. Chest 2002; 121: 1988-99.

2.- Mouy R, Fischer A, Vilmer E, Seger R, Griscelli C. Incidence, severity, and prevention of infections in chronic granulomatous disease. J Pediatr 1989; 114: 555-60.

3.- Shetty D, Giri N, González C E, Pizzo P A, Walsh T J. Invasive aspergillosis in human immunodeficiency virus-infected children. Pediatr Infect Dis J 1997; 16: 216-21.

4.- Walmsley S, Devi S, King S, Schneider R, Richardson S, Ford-Jones L. Invasive

Aspergillus infections in a pediatric hospital: a ten-year review. Pediatr Infect Dis J 1993; 12: 673-82.

5.- Denning D W. Invasive aspergillosis. Clin Infect Dis 1998, 26: 781-805.

6.- Groll A H, Jaeger G, Allendorf A, Herrmann G, Schloesser R, Von Loewenich V. Invasive pulmonary aspergillosis in a critically ill neonate: case report and review of invasive aspergillosis during the first 3 months of life. Clin Infect Dis 1998; 27: 437-52.

7.- Conesa D, Rello J, Valles J, Mariscal D, Ferreres J C. Invasive aspergillosis: a lifethreatening complication of short-term steroid treatment. Ann Pharmacother 1995; 29: $1235-7$.

8.- Ko J P, Kim D H, Shepard J A. Pulmonary aspergillosis in an immunocompetent patient. J Thorac Imaging 2002; 17: 70-3.

9.- McNeil M M, Nash S L, Hajjeh R A, Phelan M A, Conn L A, Plikaytis B D, et al. Trends in mortality due to invasive mycotic diseases in the United States, 1980-1997. Clin Infect Dis 2001; 33: 641-7.

10.- Shaw F W, Warthen H J. Aspergillosis of bone. South Med J 1936; 29: 1070-1.

11.- Bodey G. Fungal infection and fever of unknown origin in neutropenic patients. Am J Med 1986; 80: 112-9.

12.- Allo M D, Miller J, Townsend T, Tan C. Primary cutaneous aspergillosis associated with Hickman intravenous catheters. N Engl J Med 1987; 317: 1105-8.

13.- Steinbach W J. Pediatric aspergillosis. Disease and treatment differences in children. Pediatr Infect Dis J 2005; 24: 358-64.

14.- Anderson L L, Giandoni M B, Keller R A, Grabski W J. Surgical wound healing complicated by Aspergillus infection in a non-immunocompromised host. Dermatol Surg 1995; 21: 799-801.

15.- Gefter W B. The spectrum of pulmonary aspergillosis. J Thorac Imaging 1992; 7: 56-
74.

16.- Thomas K E, Owens C M, Veys P A, Novelli $\mathrm{V}$, Costoli V. The radiological spectrum of invasive aspergillosis in children: a 10-year review. Pediatr Radiol 2003; 33: 453-60.

17.- Taccone A, Occhi M, Garaventa A, Manfredini L, Viscoli C. CT of invasive pulmonary aspergillosis in children with cancer. Pediatr Radiol 1993; 23: 177-80.

18.- Caillot D, Casasnovas O, Bernard A, Couaillier J F, Durand C, Cuisenier B, et al. Improved management of invasive pulmonary aspergillosis in neutropenic patients using early thoracic computed tomographic scan and surgery. J Clin Oncol 1997; 15: 139-47.

19.- Ascioglu S, Rex J H, De Pauw B, Bennett J E, Bille J, Crokaert F, et al. Defining opportunistic invasive fungal infections in immunocompromised patients with cancer and hematopoietic stem cell transplant: an international consensus. Clin Infect Dis 2002; 34: 7-14.

20.- Fica A. Tratamiento de infecciones fúngicas sistémicas. III parte: Anfotericina B, aspectos farmacoeconómicos y decisiones terapéuticas. Rev Chil Infect 2004; 21: 317-26

21.- Hartemink K J, Paul M A, Spijkstra J J, Girbes A R, Polderman KH. Immunoparalysis as a cause for invasive aspergillosis? Intensive Care Med 2003; 29 : 2068-71.

22.- Meersseman W, Vandecasteele SJ, Wilmer A, Verbeken E, Peetermans W E, Van Wijngaerden E. Invasive aspergillosis in critically ill patients without malignancy. Am J Respir Crit Care Med 2004; 170: 621-5.

23.- Kwoun M O, Ling P R, Lydon E, Imrich A, Qu Z, Palombo J, et al. Immunologic effect of acute hyperglycemia in non diabetic rats. J Parenter Enteral Nutr 1997; 21: 91-5.

24.- Pilleux L, Ponticas M, Carrasco C, León A, Salas P, Rodríguez C, et al. Hialohifomicosis invasiva hepatoesplénica. Comunicación de un caso. Rev Méd Chile 2000; 128: 641-6.

25.- Hasejima N, Yamato K, Takezawa S, Kobayashi H, Kadoyama C. Invasive pulmonary aspergillosis associated with influenza B. Respirology 2005; 10: 116-9

26.- Siddiqui A A, Shah A A, Bashir S H. Craniocerebral aspergillosis of sinonasal origin in immunocompetent patient: clinical spectrum and outcome in 25 cases. Neurosurgery 2004; 55: 602-11.

27.- Karim M, Alam M, Shah A A, Ahmed R, Sheinkh H. Chronic invasive aspergillosis in apparently immunocompetent host. Clin Infect Dis 1997; 24: 723-33.

28.- Pannuti C, Gingrich R, Pfaller M, Kao C,
Wenzel R. Nosocomial pneumonia in patients having bone marrow transplant. Attributable mortality and risk factors. Cancer 1992; 69: 2653-62.

29.- Saugier V, Devergie A, Sulahian A, Ribaud P, Traore F, Bourdeau E. Epidemiology and diagnosis of invasive pulmonary aspergillosis in bone marrow transplant patients: Results of a 5 year retrospective study. Bone Marrow Transplant 1993; 12: 121-4.

30.- Spreadbury C, Holden D, Aufauvre-Brown A, Bainbridge B, Cohen J. Detection of Aspergillus fumigatus by polymerase chain reaction. J Clin Microbiol 1993; 31: 615-21

31.- Denning D W, Stevens D A. Antifungal and surgical treatment of invasive aspergillosis: Review of 2121 cases. Rev Infect Dis 1990; 12: 1147-201.

32.- Ribrag V, Dreyfus F, Venot A, Leblong V, Lanore J J, Varet B. Prognostic factors of invasive pulmonary aspergillosis in leukemia patients. Leuk Lymphoma 1993; 10: 317 21.

33.- Retamal C, Díaz C, Salamanca L, Ferrada L, Oro R. Aspergilosis pulmonar en Chile enfoque inmunológico. Bol Micol 1984; 2: 11-6.

34.- Herbrecht R, Denning D W, Patterson T F, Bennett J E, Greene R E, Oestmann J W, et al. Invasive Fungal Infections Group of the European Organization for Research and Treatment of Cancer and the Global Aspergillus Study Group. Voriconazole versus amphotericin B for primary therapy of invasive aspergillosis. N Engl J Med 2002; 347: 408-15.

35.- Bidart T. Rol de voriconazol y caspofungina en terapia antifúngica. Rev Chil Infect 2004; 21 (Supl 1): S13-S19.

36.- Maertens J, Raad I, Petrikkos G, Boogaerts M, Selleslag D, Petersen F, et al. for the Caspofungin Salvage Aspergillosis Study Group. Efficacy and safety of caspofungin for treatment of invasive aspergillosis in patients refractory to or intolerant of conventional antifungal therapy. Clin Infect Dis 2004; 39: 1563-71.

37.- Walsh T J, Adamson P C, Seibel N L, Flynn P M, Neely M N, Schwartz C, et al. Pharmacokinetics, safety, and tolerability of caspofungin in children and adolescents. Antimicrob Agents Chemother 2005; 49: 4536-45.

38.- Walsh T J, Karlsson M O, Driscoll T, Argüedas A, Adamson P, Saez-Llorens X, et al. Pharmacokinetics and safety of voriconazole in children after single or multiple-dose administration. Antimicrob Agents Chemother 2005; 48: 2166-72. 